\title{
Toward interpretable, wide field-of-view transmission electron microscopy techniques for imaging light atoms
}

\author{
Hamish Brown $^{1}$ and Jim Ciston ${ }^{2}$ \\ ${ }^{1}$ The University of Melbourne, Parkville, Victoria, Australia, ${ }^{2}$ UC Berkeley, California, United States
}

Transmission electron microscopy (TEM) is a technique of choice for Angstrom resolution, or better, imaging of samples for materials science and biology. A TEM instrument is typically operated in conventional TEM (CTEM) mode, see Fig. 1(a) where an image is formed of the electron wave after passing through the specimen, or scanning TEM (STEM), were the proportion of electrons scattered to a diffraction plane detector is measured as a focused probe is rastered over the specimen. In materials science CTEM is favoured for in-situ studies where time resolved imaging over wide fields-of-view is required, whilst STEM typically results in somewhat more directly interpretable images in the presence of strong electron multiple scattering in crystalline materials. Annular bright-field (ABF) STEM, where the diffraction plane detector covers the set of angles $-\alpha / 2<\beta<a$ where $a$ is the probe convergence semi-angle, is a STEM technique favoured for its ability to produce interpretable images of light and heavy atoms in thick crystalline samples [1]. A technique combining the ability of CTEM to simultaneously image wide fields-of-view but with the ability to form more directly interpretable images of light and heavy atoms in crystalline materials as in STEM is desirable.

The principle of STEM-CTEM reciprocity, first discussed by Pogany and Turner in 1968, suggests a way of achieving this "best of both worlds" and holds that for any STEM result a similar CTEM result should be achieved if the roles of the detector and the illumination are reciprocal [2]. In particular, a CTEM experiment where the beam is precessed through a solid cone corresponding to the angular range of a STEM detector will produce equivalent results. To replicate ABF STEM contrast in a CTEM setup we precessed the beam in a cone just inside the radius of a post specimen objective aperture, a technique known as dynamic hollow-cone illumination (DHCI -TEM) [3], see Fig. 1(c).

With a [001] oriented SrTiO3 crystal as our test specimen we demonstrate atomic resolution imaging using the DCHI-TEM technique, shown in Fig. 2(a). A unit cell average of the image (labelled UCA) is inset bottom right in Fig. 2(a) and the atomic columns are visible as black contrast. A simulation, also inset in Fig 2. (a), reveals that the more prominent black columns are indeed $\mathrm{Sr}$ and $\mathrm{Ti}$ and that the features in between these columns are the lighter oxygen columns [4]. A CTEM image of a similar region, Fig. 2(b) does not reveal the oxygen columns at this focus though the signal to noise is superior to DHCI-TEM. In this talk we provide a detailed comparison of DHCI TEM to ABF STEM and CTEM and future research directions that might lead to CTEM approaches that provide both improved interpretability without sacrificing contrast relative to standard CTEM [5]. 
(a) CTEM

(b) STEM

(c) DHCI TEM

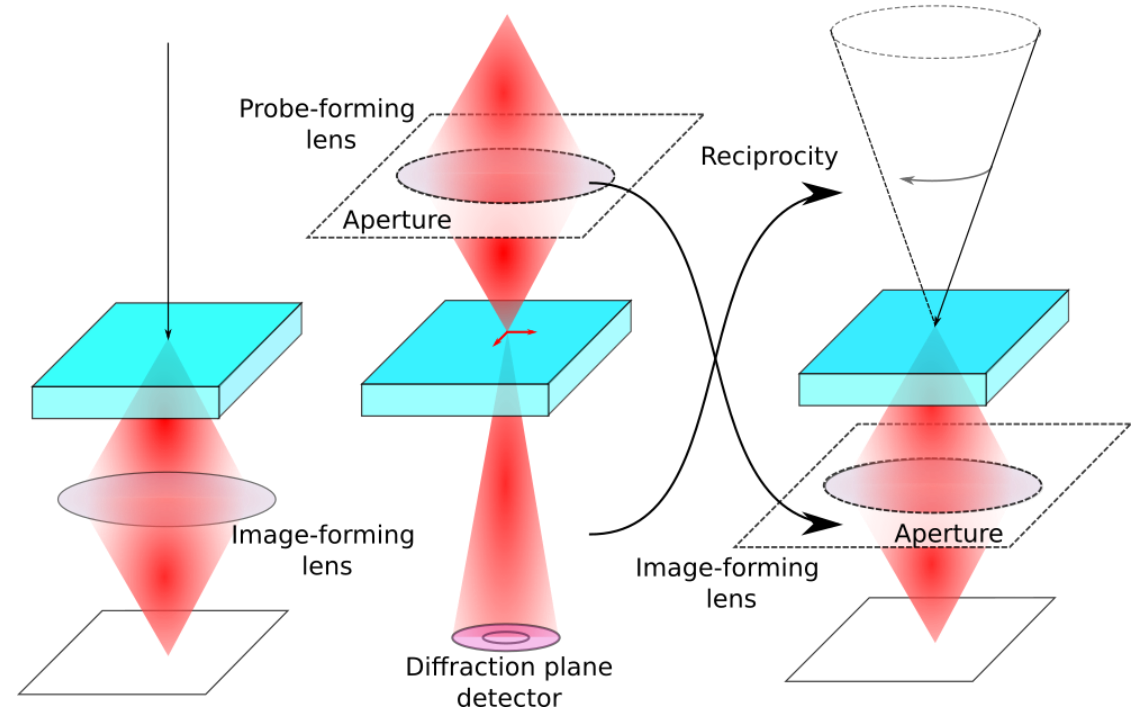

Figure 1.Sketches of the experimental setup of conventional TEM (CTEM), scanning TEM (STEM) and dynamic hollowcone illumination (DHCI ) TEM.

(a) $\mathrm{DHCl} \mathrm{TEM}$

(b) CTEM

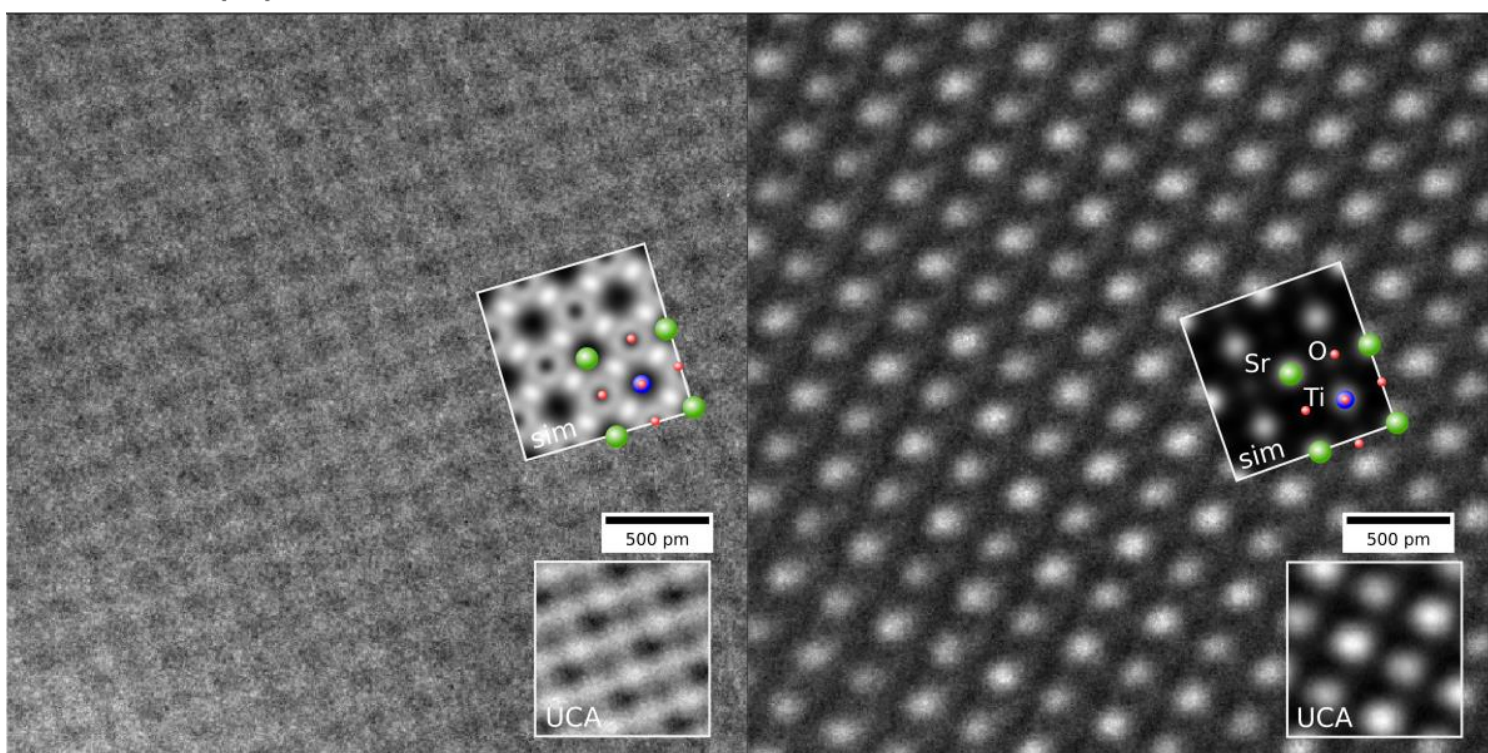

Figure 2. Imaging a [001] SrTiO3 crystal with (a) DHCI TEM and (b) CTEM. The unit cell average (UCA) of the DHCI TEM result reveals unambiguously the presence of oxygen atoms alongside the heavier atoms in the unit cell whilst the CTEM image has higher contrast but oxygen atoms are not visible for the microscope parameters here.

\section{References}

[1] Findlay, S. D., et al. "Robust atomic resolution imaging of light elements using scanning transmission electron microscopy." Applied Physics Letters 95.19 (2009): 191913.

[2] Pogany, A. P., and P. S. Turner. "Reciprocity in electron diffraction and microscopy." Acta Crystallographica Section A: Crystal Physics, Diffraction, Theoretical and General Crystallography 24.1 (1968): 103-109. 
[3] Kunath, W., F. Zemlin, and K. Weiss. "Apodization in phase-contrast electron microscopy realized with hollow-cone illumination." Ultramicroscopy 16.2 (1985): 123-138.

[4] Brown, Hamish G., and Jim Ciston. "Atomic Resolution Imaging of Light Elements in a Crystalline Environment using Dynamic Hollow-Cone Illumination Transmission Electron Microscopy." Microscopy and Microanalysis 26.4 (2020): 623-629.

[5] Work at the Molecular Foundry was supported by the Office of Science, Office of Basic Energy Sciences, of the U.S. Department of Energy under Contract No. DE-AC02-05CH11231. HGB and JC acknowledge additional support from the Presidential Early Career Award for Scientists and Engineers (PECASE) through the U.S. Department of Energy. The authors acknowledge sample preparation support from Thermo Fisher Scientific. Helpful discussions with Dr. Alex Eggeman of the University of Manchester are also acknowledged. 